\title{
A Method to Transit the Rotor-to-Stator Rubbing to Normal Motion Using the Phase Characteristic
}

\author{
Jieqiong Xu, ${ }^{1}$ Qunhong Li, ${ }^{1}$ and Shimin Wang ${ }^{2}$ \\ ${ }^{1}$ College of Mathematics and Information Science, Guangxi University, Nanning 530004, China \\ ${ }^{2}$ The Research Lab of Dynamics and Control, Beijing University of Aeronautics and Astronautics, Beijing 100091, China \\ Correspondence should be addressed to Jieqiong Xu; clh4@163.com
}

Received 26 January 2014; Revised 15 July 2014; Accepted 23 July 2014; Published 11 August 2014

Academic Editor: Michael A. Radin

Copyright (c) 2014 Jieqiong Xu et al. This is an open access article distributed under the Creative Commons Attribution License, which permits unrestricted use, distribution, and reproduction in any medium, provided the original work is properly cited.

\begin{abstract}
A method is proposed to transit the rotor-to-stator rubbing to no-rub motion through active auxiliary bearing. The key point of this technique is to express the attractive domain of no-rub motion based on the phase characteristic and to represent the desired status. The feedback actuation is applied by an active auxiliary bearing to drive the rotor approaching the desired status. After that, the control actuation is turned off. Although the desired status is still in rubbing, it is in the attractive domain of no-rub motion, and the response of the rotor is automatically attracted to no-rub motion.
\end{abstract}

\section{Introduction}

Rubbing between the rotor and the stator is a serious malfunction, which frequently happens in the operation of a rotating machine. It may lead to heavy damage of the machine or even to complete destruction. Therefore, it is necessary to understand the dynamical behavior of the rotor-to-stator rubbing and to control it. Chu and Zhang [1] investigated the reliability of the rotor system with rubbing and found out four different scenarios to and out of chaos. Some publications researched the whirling motion of full annular rotor rubbing, for example, [2, 3]. Karpenko et al. [4] described the experimental verification of a nonlinear Jeffcott rotor model with a preloaded snubber ring. Popprath and Ecker [5] presented a mathematical model to investigate the dynamics of a Jeffcott rotor having intermittent contact with a stator and exhibited rich dynamical behavior by numerical results. Jiang [6] studied the global response characteristics of a piecewise smooth dynamical system with contact analytically.

For controlling the rotor-to-stator rubbing, many works investigated control forces acting directly on the rotor to minimize rotor vibration, for example, [7-12]. A control strategy using an active auxiliary bearing was proposed, in which actuation was on the auxiliary bearing and thereby indirectly on the rotor. Compared with the conventional auxiliary bearing, the active auxiliary bearing has the following advantages [13]: it not only limits the large response amplitude of the rotor for preventing the rotor/blades and the casing/seals from direct contact, but it also effectively reduces the rubbing severity, and it especially avoids the occurrence of the destructive rubbing instabilities. Additionally, the capability of existing auxiliary bearings can be well extended from this concept by introducing active control. Ginzinger and Ulbrich [13] developed a two-phase control strategy to control a rubbing rotor applying an active auxiliary bearing. Chavez et al. [14] used sliding control and cross coupled feedback in a rotor system driven by a power limited motor to reduce the impact forces and to decrease the lateral and torsional vibrations. Ginzinger and Ulbrich [15] presented a framework for the development of a feedback controller for an active auxiliary bearing. Cade et al. [16] considered an active auxiliary bearing system with a control strategy designed to limit the trapped contact modes in a rotor/magnetic bearing system. Because the auxiliary bearing exerts control forces on the rotating system only when rotor establishes contact with the auxiliary bearing, [13-16] dealt with the problem of rotor-to-stator rubbing mainly by minimizing contact force or bringing system into a stable full annular rub. 
Usually the state variables are chosen as displacement and velocity; however, amplitude and phase are also key variables for describing the vibration. In our previous work [17], a method was proposed to reduce the vibration amplitude at resonance by modulating the phase relations between excitement and response. Also, the phase characteristic of a disk rubbing with a ring supported elastically was used to explain the mechanism of stiffness increase phenomenon [18]. In this work, based on the phase characteristic, a control scheme is proposed to eliminate the rubbing of the rotor system by active auxiliary bearing. In the following section, the model of the piecewise smooth rotor/stator with a Jeffcott rotor and an active auxiliary bearing is introduced. In Section 3, the phase characteristic of the rubbing rotor system is analyzed. In Section 4, the corresponding control method is presented. In Section 5, the method is numerically simulated.

\section{Mathematical Model}

The rotor-to-stator system with a Jeffcott rotor and an active auxiliary bearing is shown in Figure 1(a). A disk with mass $m$ is mounted at the midpoint of the weightless shaft with total stiffness $k_{r}$, viscous $c$, and rotating at a constant speed of $\omega$. The mass center of the rotor is located at a distance $\widetilde{e}$ from its geometrical center. The auxiliary bearing is considered to be rigid and modeled as the radial springs with stiffness $k_{s}$. The clearance between the rotor and the auxiliary bearing is denoted by $\gamma$. For directly expressing the phase difference between the excitement (the rotating motion) and the response (the whirling motion), a polar coordinate system is chosen, shown as Figure 1(b). The center of the auxiliary bearing is assumed as the origin of the coordinates. The current position of the rotor $O_{r}$ is given by the radius $\tilde{\rho}$ and the angle $\psi$. The phase angle of the imbalance force is $\varphi=\omega t$. When $\tilde{\rho} \geq \gamma$, the normal contact force and the friction force between the rotor and the auxiliary bearing are $F_{N}$ and $F_{T}=$ $\mu F_{N}$, respectively, where $\mu$ is the friction coefficient.

The equations that govern the motion of the rotor-tostator system in polar coordinate form can be formulated into nondimensional form as

$$
\begin{gathered}
\ddot{\rho}+\nu \dot{\rho}+\rho-\rho \dot{\psi}^{2}+\Theta f_{N}-e \omega^{2} \cos (\omega \tau-\psi)=0, \\
\rho \ddot{\psi}+2 \dot{\rho} \dot{\psi}+\nu \rho \dot{\psi}+\Theta \mu f_{N}-e \omega^{2} \sin (\omega \tau-\psi)=0, \\
f_{N}=k(\rho-1),
\end{gathered}
$$

where the dot denotes the derivatives with respect to nondimensional time $\tau$. We define $\Theta=1$ if $\rho \geq 1$ and $\Theta=0$, if $\rho<1$. The nondimensional parameters and variables are listed as follows:

$$
\begin{gathered}
\omega_{n}^{2}=\frac{k_{r}}{m}, \quad \tau=\omega_{n} t, \quad \rho=\frac{\tilde{\rho}}{\gamma}, \quad \nu=\frac{c}{2 \sqrt{k_{r} m}}, \\
e=\frac{\tilde{e}}{\gamma}, \quad f_{N}=\frac{F_{N}}{k_{r} \gamma}, \quad k=\frac{k_{s}}{k_{r}} .
\end{gathered}
$$

\section{Phase Characteristics of Rubbing Motion}

In this section, the characteristics of the phase difference between the excitement force and the response $\varphi-\psi$ are analyzed. It is noticed that the $\varphi-\psi$ represents $\varphi-\psi$ or $\bmod (\varphi-\psi, 2 \pi)$, which is in the range $[0,2 \pi]$.

The rotor/stator system governed by (1) has a steadystate periodic solution that has a constant amplitude and a frequency equal to the rotating speed of the rotor. The solution is corresponding to $\ddot{\rho}=\dot{\rho}=\ddot{\psi}=0$ and $\dot{\psi}=\dot{\varphi}=\omega$; substituting this specific solution into (1) yields

$$
\begin{gathered}
\rho-\rho \dot{\psi}^{2}+\Theta k(\rho-1)-e \omega^{2} \cos (\varphi-\psi)=0, \\
\nu \rho \dot{\psi}+\Theta \mu k(\rho-1)-e \omega^{2} \sin (\varphi-\psi)=0 .
\end{gathered}
$$

From (3), the radial amplitude $\rho$ and the phase difference $\varphi-\psi$ are obtianed, when $\Theta=0$,

$$
\begin{gathered}
\left(\rho-\rho \omega^{2}\right)^{2}+(\nu \rho \omega)^{2}=\left(e \omega^{2}\right)^{2} \\
\tan (\varphi-\psi)=\frac{\nu \omega}{1-\omega^{2}} .
\end{gathered}
$$

When $\Theta=1$,

$$
\begin{aligned}
& \left(\rho-\rho \omega^{2}+k \rho-k\right)^{2}+(\nu \rho \omega+\mu k \rho-\mu k)^{2}=\left(e \omega^{2}\right)^{2} \\
& e \omega^{2}\left[(\nu \omega+\mu k) \cos (\varphi-\psi)-\left(1+k-\omega^{2}\right) \sin (\varphi-\psi)\right] \\
& \quad=k\left(\mu-\mu \omega^{2}-\nu \omega\right) .
\end{aligned}
$$

The amplitude and the phase difference are expressed as a function of the rotor speed $\omega$ in (4) and (5). In (4), the nondimensional viscous $v$ is small; it is obvious that the phase difference $\varphi-\psi$ approaches to $\pi$ with the increase of the rotor speed $\omega$ if there exists no-rub solution when $\omega>1$. For synchronous full annular rub solution, the phase difference $\varphi-\psi$ is less than $\pi / 2$, which was explained in [18]. As an example, the phase difference and radial displacement depending on the rotor speed are numerically plotted according to (4) and (5) in Figures 2(a) and 2(b), respectively, for $\nu=0.1, \mu=0.06, e=0.2$, and $k=8$.

In Figure 2(a), the contact does not happen along the curve denoted by 1 . The rotor first establishes contact with the bearing at the rotor speed $\omega=0.925$ marked as 2 where the curve has its first kink. It is found that when the contact occurs, the phase difference $\varphi-\psi$ is less than $\pi / 2$ until the speed approaches $\omega=2.321$ marked by 3. At the point 3 , an infinitesimal increase in rotor speed causes the phase difference to jump to point 5 , about $\pi$. Then, the phase difference is kept along the curve marked by $5-6$. When the rotor speed is decreased, the phase difference follows the curve 6-5-4-2-1. A jump occurs at the point marked by 4 , where the phase difference becomes less than $\pi / 2$ again. The corresponding radial amplitude diagrams are plotted in Figure 2(b). It can be seen that the amplitude jumps at the same speeds as the phase difference. Particularly, it is noticed that no-rub solution and rubbing solution coexist between the rotor speed $\omega=1.103$ and $\omega=2.321$. The phase difference 


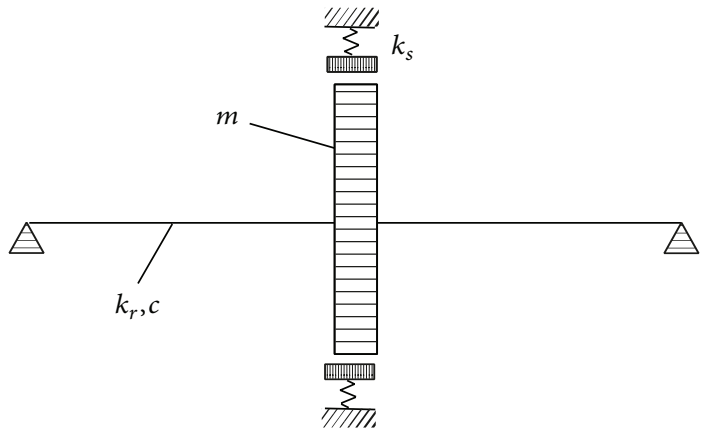

(a)

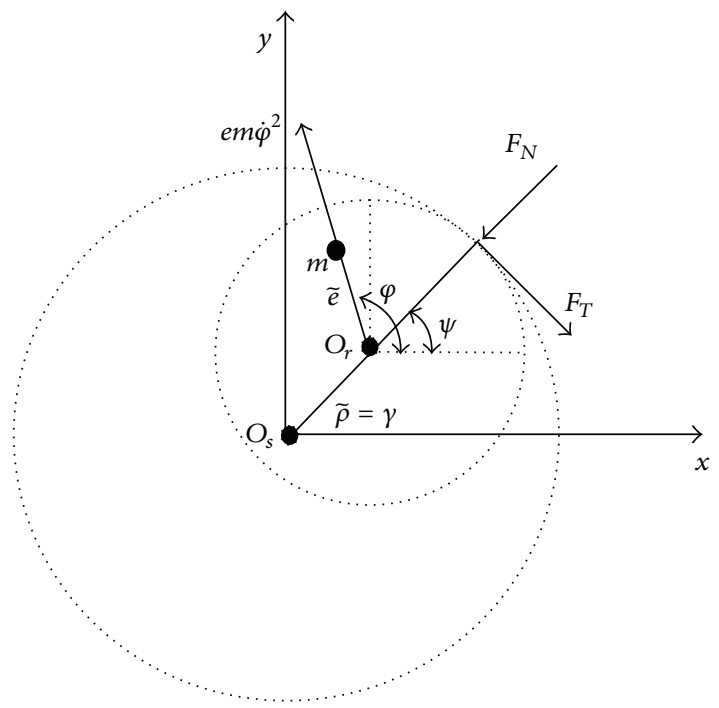

(b)

Figure 1: (a) The schematic plot of the rotor-to-stator system; (b) the section view on the plane of the rotor and the stator ring.

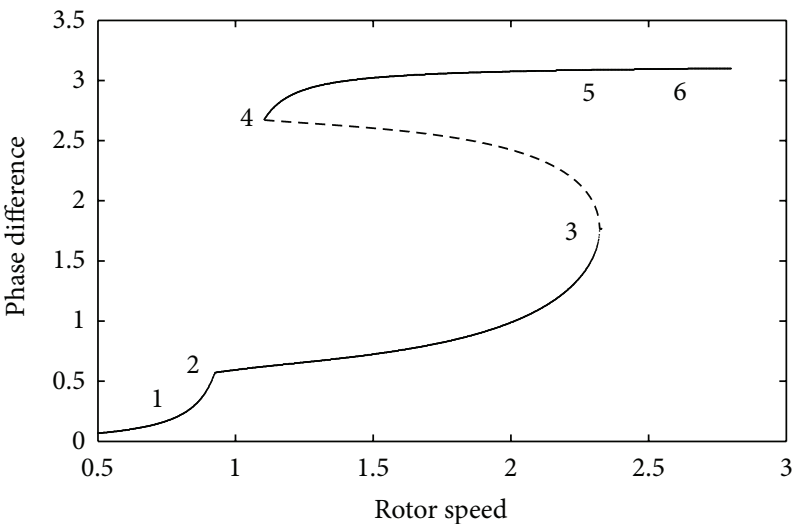

(a)

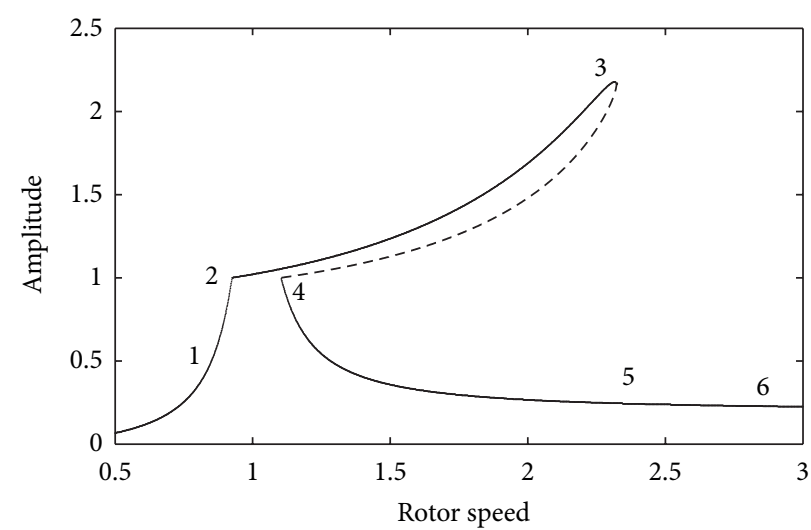

(b)

FIgURe 2: (a) Phase difference shift depending on rotor speed; (b) radial displacement amplitude depending on rotor speed.

of the no-rub solution is about $\pi$ while that of the rubbing solution is less than $\pi / 2$, and the dashed curve represents unstable solution between the no-rub and rubbing solutions, which is important for determining the attractive domain of the no-rub motion. $(\rho, \dot{\rho}, \psi, \dot{\psi})$ represents the position of rotor center, the phase difference $\varphi-\psi$ should near $\pi / 2$ if $(\rho, \dot{\rho}, \psi, \dot{\psi})$ is in the attractive domain of the rubbing motion while the phase difference $\varphi-\psi$ should near $\pi$ if $(\rho, \dot{\rho}, \psi, \dot{\psi})$ is in the attractive domain of the no-rub motion.

In certain parameters, the synchronous full annular rub solutions become unstable; then partial rubs and dry whip may occur in the system. As an example, the bifurcation diagrams of the amplitude and the phase difference against rotor speed for parameters $v=0.1, \mu=0.06, e=0.2$, and $k=8$ are plotted in Figure 3. The scenario of rotor responses with the increase of the rotating speed is no-rub synchronous whirl $\rightarrow$ a synchronous full annular rub $\rightarrow$ a partial rub with forward whirl $\rightarrow$ a partial rub with backward whirl $\rightarrow$ dry whip as shown in Figure 3.

To eliminate these rubs with real time control methods, the difficulty is that the auxiliary bearing exerts action on the rotor only if contact is kept, while the interaction between the bearing and the rotor will induce the stiffness increase; therefore, some previous control strategies were mainly configured to minimize contact force or to control the system into a stable full annular rub. In other words, the motion is still in rubbing contact. Indeed, there exist stable no-rub motion and rub motion at the same rotor speed. In this work, a control approach is developed to transit the complex behaviors in rubs to no-rub motion based on the phase characteristic. 


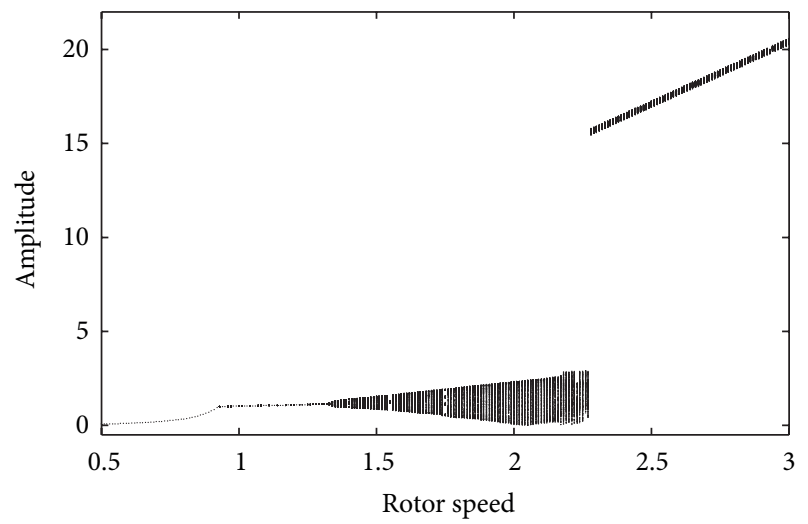

(a)

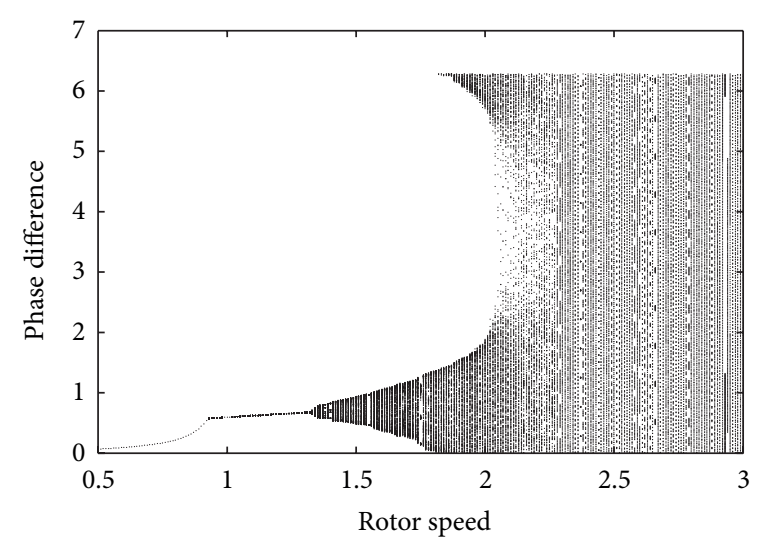

(b)

FIGURE 3: Bifurcation diagrams of (a) radial displacement amplitude and (b) phase difference.

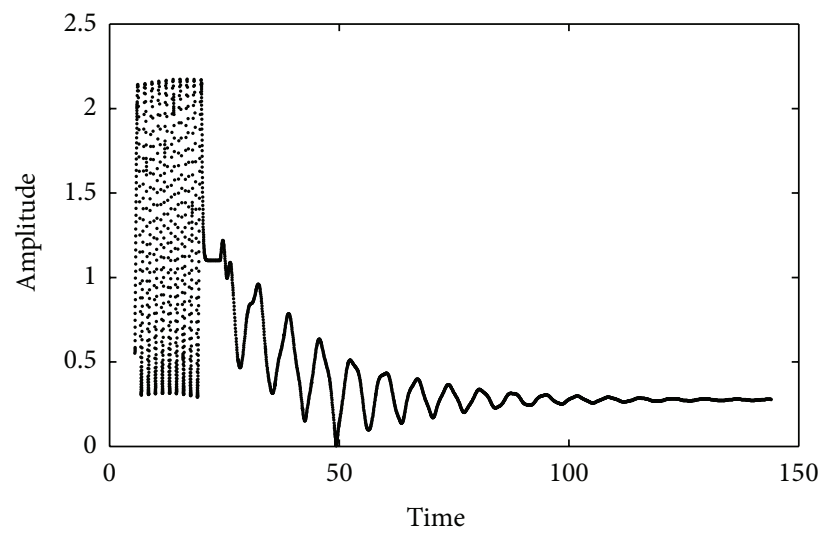

(a)

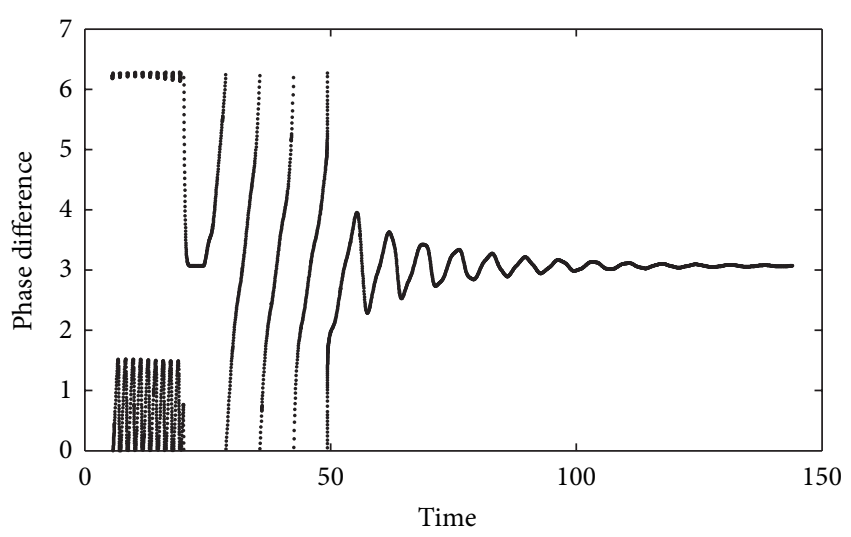

(b)

FIGURE 4: Control result at $\omega=1.9$. Time history of (a) the radial displacement amplitude and (b) phase difference.

\section{Controller Design}

The control strategy in this paper consists of two steps: at first, when the rotor is rubbing with the active auxiliary bearing, the feedback actuation is applied by the active auxiliary bearing to drive rotor to the desired status. Although the desired status is not a no rub vibration with small amplitude, it is in the attractive domain of no rub motion; then, the feedback actuation is turned off because the desired status can be transited to the no-rub motion and the no-rub motion is maintained without control. Therefore, the key point of this control strategy is to find the attractive domain of no-rub motion such that the desired status can be determined.

The phase characteristic analyzed in Section 3 is used to find the attractive domain of no-rub motion. Although the exact boundary of the attractive domain of no-rub motion is not obtained, the approximate one is enough to determine the desired status.

The desired nondimensional radial displacement must be greater than 1 for that auxiliary bearing to exert control action on the rotor. According to the analysis in Section 3, the unstable solution separates the no-rub and rubbing solutions, and the phase difference of no-rub motion is near $\pi$, so the desired phase difference is in neighborhood of $\pi$.

For the convenience, we introduce $x_{1}=\rho, x_{2}=\dot{\rho}, x_{3}=\psi$, $x_{4}=\dot{\psi}$, and $X(t)=\left\{x_{1}, x_{2}, x_{3}, x_{4}\right\}$. When $\Theta=1,(1)$ can be described as

$$
\begin{aligned}
& \dot{x}_{1}=x_{2}, \\
& \dot{x}_{2}=-x_{1}-v x_{2}+x_{1} x_{4}^{2}-k\left(x_{1}-1\right)+e \omega^{2} \cos \left(\omega t-x_{3}\right), \\
& \dot{x}_{3}=x_{4}, \\
& \dot{x}_{4}=\frac{\left[-2 x_{2} x_{4}-v x_{1} x_{4}-\mu k\left(x_{1}-1\right)+e \omega^{2} \sin \left(\omega t-x_{3}\right)\right]}{x_{1}} .
\end{aligned}
$$

The desired status is given by $\left\{x_{1 d}, x_{2 d}, x_{3 d}, x_{4 d}\right\}$, where $x_{1 d}$ is the desired radial displacement, which is an adequate magnitude and is greater than $1, x_{2 d}=\dot{x}_{1 d}, x_{3 d}$ is the desired phase angle, and $x_{4 d}=\dot{x}_{3 d}$. let $y_{3 d}=\omega t-x_{3 d}$ be the desired phase difference and $y_{4 d}=\omega-x_{4 d}$. Based on the phase characteristic in Section 3, the desired phase difference $y_{3 d}=\omega t-x_{3 d}$ should be in neighborhood of $\pi$. The first 


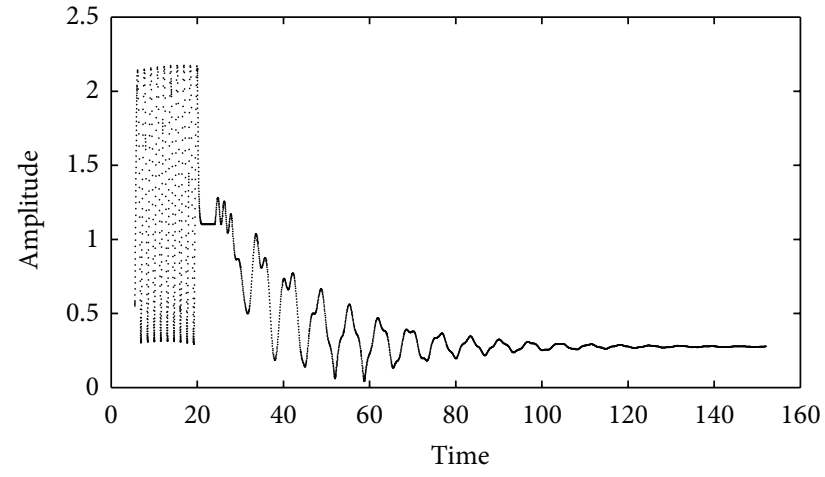

(a)

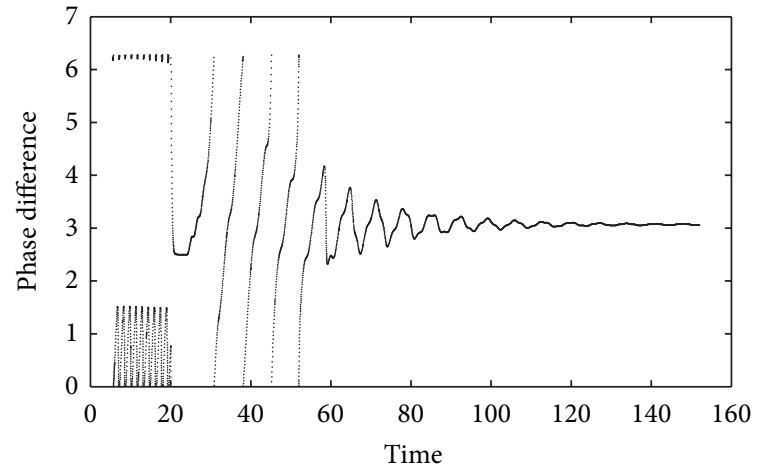

(b)

FIGURE 5: Control result at $\omega=1.9$. Time history of (a) the radial displacement amplitude and (b) phase difference.

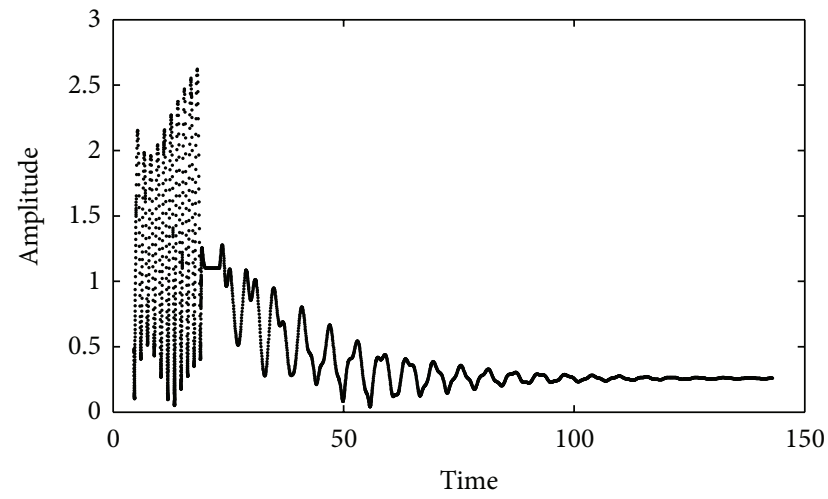

(a)

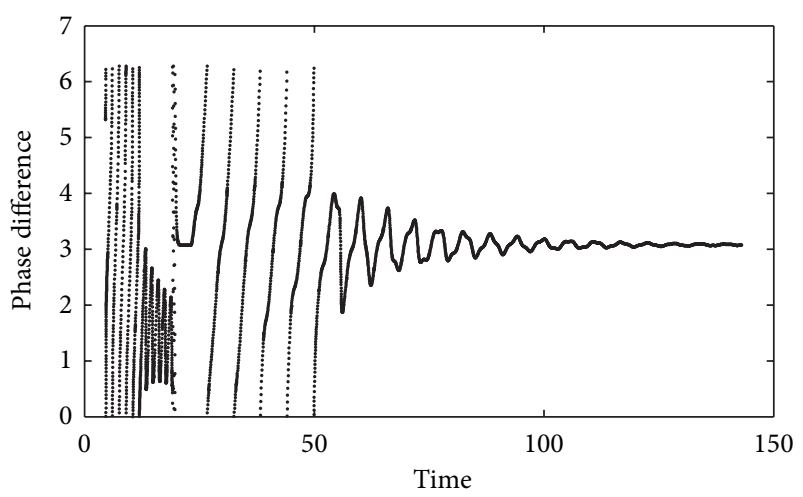

(b)

FIgure 6: Control result at $\omega=2.1$. Time history of (a) the radial displacement amplitude and (b) phase difference.

step of control strategy is to choose an appropriate controller $U=\left\{u_{1}, u_{2}\right\}$ that satisfies

$$
\begin{gathered}
\lim _{t \rightarrow \infty}\left|x_{1}(t)-x_{1 d}\right|=0, \\
\lim _{t \rightarrow \infty}\left|x_{3}(t)-\omega t+y_{3 d}\right|=0 .
\end{gathered}
$$

The control system can be described as

$$
\begin{aligned}
\dot{x}_{1}= & x_{2}, \\
\dot{x}_{2}= & -x_{1}-v x_{2}+x_{1} x_{4}^{2}-k\left(x_{1}-1\right)+e \omega^{2} \cos \left(\omega t-x_{3}\right) \\
& +u_{1}, \\
\dot{x}_{3}= & x_{4}, \\
\dot{x}_{4}= & {\left[-2 x_{2} x_{4}-v x_{1} x_{4}-\mu k\left(x_{1}-1\right)\right.} \\
& \left.\quad+e \omega^{2} \sin \left(\omega t-x_{3}\right)+u_{2}\right] \times\left(x_{1}\right)^{-1} .
\end{aligned}
$$

Let

$$
\begin{aligned}
& e_{1}=x_{1}-x_{1 d}, \\
& e_{2}=x_{2}-\dot{x}_{1 d}, \\
& e_{3}=x_{3}-\omega t+y_{3 d}, \\
& e_{4}=x_{4}-\omega+\dot{y}_{3 d} .
\end{aligned}
$$

The error equations become

$$
\begin{aligned}
\dot{e}_{1}= & e_{2}, \\
\dot{e}_{2}= & -\left(e_{1}+x_{1 d}\right)-v e_{2}+\left(e_{1}+x_{1 d}\right)\left(e_{4}+\omega\right)^{2} \\
& -k\left(e_{1}+x_{1 d}-1\right)+e \omega^{2} \cos \left(y_{3 d}-e_{3}\right)+u_{1}, \\
\dot{e}_{3}= & e_{4}, \\
\dot{e}_{4}= & \left(-2 e_{2}\left(e_{4}+\omega\right)-v\left(e_{1}+x_{1 d}\right)\left(e_{4}+\omega\right)\right. \\
& \left.-\mu k\left(e_{1}+x_{1 d}-1\right)+e \omega^{2} \sin \left(y_{3 d}-e_{3}\right)+u_{2}\right) \\
& \times\left(e_{1}+x_{1 d}\right)^{-1} .
\end{aligned}
$$




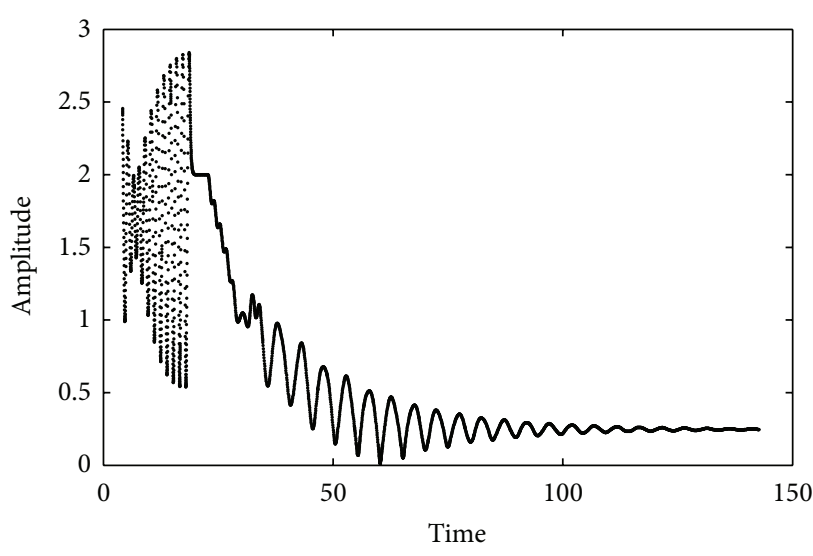

(a)

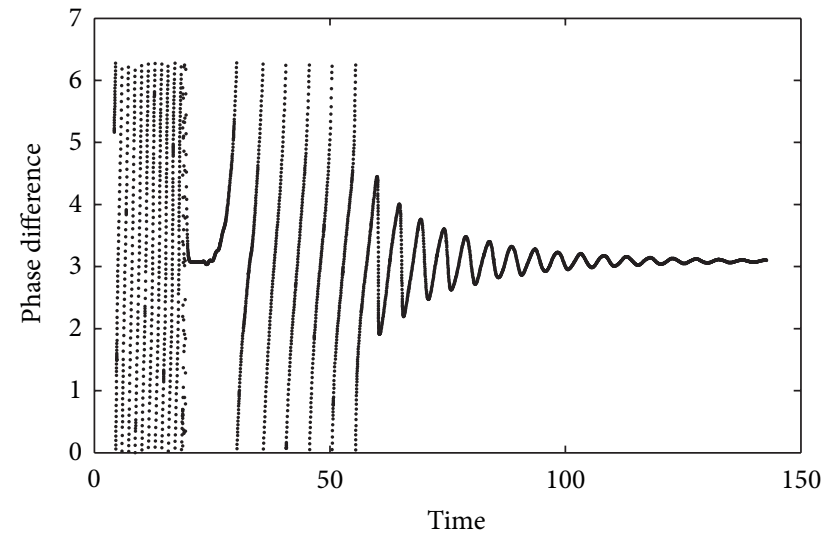

(b)

Figure 7: Control result at $\omega=2.28$. Time history of (a) the radial displacement amplitude and (b) phase difference.

To diminish the error equations (10), the action of the controller is proposed as

$$
\begin{aligned}
u_{1}= & \left(e_{1}+x_{1 d}\right)+v e_{2}-\left(e_{1}+x_{1 d}\right)\left(e_{4}+\omega\right)^{2} \\
& +k\left(e_{1}+x_{1 d}-1\right)-e \omega^{2} \cos \left(y_{3 d}-e_{3}\right) \\
& -\left(a_{1} e_{1}+b_{1} e_{2}\right), \\
u_{2}= & 2 e_{2}\left(e_{4}+\omega\right)+v\left(e_{1}+x_{1 d}\right)\left(e_{4}+\omega\right)+\mu k\left(e_{1}+x_{1 d}-1\right) \\
& -e \omega^{2} \sin \left(y_{3 d}-e_{3}\right)-\left(e_{1}+x_{1 d}\right)\left(a_{2} e_{3}+b_{2} e_{4}\right) .
\end{aligned}
$$

The errors of (10) are governed mainly by the design parameters $\left(a_{1}, b_{1}, a_{2}, b_{2}\right)$, which can be determined by letting all eigenvalues of Jacobian of (10) have negative real parts. The rotor approaches the desired status for suitable values of $\left(a_{1}, b_{1}, a_{2}, b_{2}\right)$. For example, in the next section, the values $\left(a_{1}, b_{1}, a_{2}, b_{2}\right)$ in $(10)$ are set as $(6,8,6,8)$ to guarantee that the eigenvalues are with negative real parts and that the trajectory reaches the desired status.

\section{Numerical Simulations}

In the following, numerical simulations are demonstrated to show the practical feasibility of this idea for parameters $v=$ $0.1, \mu=0.06, e=0.2$, and $k=8$.

For rotor speed $\omega=1.9$, the response of the rotor is a partial rub with forward whirl as shown in Figure 3. The control results in Figure 4 show the amplitude of the rotor $\rho$ and the phase difference $\omega t-\psi$ under the driving frequency at $\omega=1.9$. The jump in figure is caused by mod $(\varphi-\psi, 2 \pi)$. The aim is to eliminate the partial rub between the rotor and stator. According to the phase characteristic, $y_{3 d}$, should approach $\pi$. The desired controlled position and the values $\left(a_{1}, b_{1}, a_{2}, b_{2}\right)$ in $(10)$ are set as $(1.1,0, \omega t-3.07, \omega)$ and $(6,8,6,8)$. The control input is activated at $t=20$, and after a short transition the rotor reaches the desired position $(1.1,0, \omega t-3.07, \omega)$. At this time, the control input is inactivated, and after a short time the response of the rotor is stabilized to no-rub motion itself. The partial rub with forward whirl of rotor is stabilized to no-rub motion successfully.

From the above analysis in Section 4, it is known that the desired phase difference can be chosen in neighborhood of $\pi$. For the same rotor speed $\omega=1.9$, the desired controlled position is set as $(1.1,0, \omega t-2.5, \omega)$ and the other values are not changed; the partial rub with forward whirl of rotor is also stabilized to no-rub motion successfully as shown in Figure 5. It is shown that the desired phase difference has a wide tolerance.

For rotor speed, $\omega=2.1$, the response of rotor is a partial rub with backward whirl as shown in Figure 3. The desired controlled position and the values $\left(a_{1}, b_{1}, a_{2}, b_{2}\right)$ in (9) are set as $(1.1,0, \omega t-3.07, \omega)$ and $(6,8,6,8)$. The control input is activated at $t=20$, and after a short transition the rotor reaches the desired position $(1.1,0, \omega t-3.07, \omega)$. Once the rotor enters the attractive domain, the partial rub with backward whirl can be stabilized to no-rub motion even without control. The results are shown in Figure 6.

For rotor speed $\omega=2.28$, the response of rotor is dry whip as shown in Figure 3. There is a period of transient before the response of rotor becomes dry whip, and the control input is activated in this period of time. Using the control strategy proposed in this paper, the dry whip is stabilized to no-rub motion as shown in Figure 7. In this case, the desired controlled position and the values $\left(a_{1}, b_{1}, a_{2}, b_{2}\right)$ in (9) are set as $(2,0, \omega t-3.07, \omega)$ and $(6,8,6,8)$.

\section{Conclusions}

This paper proposes a method to control rotor-to-stator rubbing based on the phase characteristics of the rotor. It has three advantages: firstly, the method eliminates the rubbing between the rotor and the stator. Secondly, the control input need not be activated all the time and can be turned off when the trajectory enters the attractive domain of the norub motion; then, the response of the rotor is attracted to no-rub motion automatically. Finally, because the phase 
characteristic is invariant, the control strategy is the same for various rubs.

\section{Conflict of Interests}

The authors declare that there is no conflict of interests regarding the publication of this paper.

\section{Acknowledgments}

This work is supported by the Project sponsored by the National Natural Science Foundation of China (no. 11372077), the Guangxi University Foundation sponsored by the Comprehensive Strength Enhancement Project of Midwest universities, the Guangxi Natural Science Foundation (no.2013GXNSFAA019017 and no.2014GXNSFBA118024), and the Scientific Research Foundation of Guangxi University (no. XBZ120251).

\section{References}

[1] F. Chu and Z. Zhang, "Bifurcation and chaos in a rub-impact Jeffcott rotor system," Journal of Sound and Vibration, vol. 210, no. 1, pp. 1-18, 1998.

[2] J. J. Yu, P. Goldman, D. E. Bently, and A. Muzynska, "Rotor/seal experimental and analytical study on full annular rub," Journal of Engineering for Gas Turbines and Power, vol. 124, no. 2, pp. 340-350, 2002.

[3] Y. S. Choi, "Investigation on the whirling motion of full annular rotor rub," Journal of Sound and Vibration, vol. 258, no. 1, pp. 191-198, 2002.

[4] E. V. Karpenko, M. Wiercigroch, E. E. Pavlovskaia, and R. D. Neilson, "Experimental verification of Jeffcott rotor model with preloaded snubber ring," Journal of Sound and Vibration, vol. 298, no. 4-5, pp. 907-917, 2006.

[5] S. Popprath and H. Ecker, "Nonlinear dynamics of a rotor contacting an elastically suspended stator," Journal of Sound and Vibration, vol. 308, no. 3-5, pp. 767-784, 2007.

[6] J. Jiang, "Determination of the global responses characteristics of a piecewise smooth dynamical system with contact," Nonlinear Dynamics, vol. 57, no. 3, pp. 351-361, 2009.

[7] P. E. Allaire, D. W. Lewis, and J. D. Knight, "Active vibration control of a single mass rotor on flexible supports," Journal of the Franklin Institute, vol. 315, no. 3, pp. 211-222, 1983.

[8] P. Tang, A. B. Palazzolo, A. F. Kascak, and G. T. Montague, "Electromechanical modeling of hybrid piezohydraulic actuator system for active vibration control," Journal of Dynamic Systems, Measurement and Control, Transactions of the ASME, vol. 119, no. 1, pp. 10-18, 1997.

[9] M. O. T. Cole and P. S. Keogh, "Asynchronous periodic contact modes for rotor vibration within an annular clearance," Proceedings of the Institution of Mechanical Engineers C: Journal of Mechanical Engineering Science, vol. 217, no. 10, pp. 1101-1116, 2003.

[10] M. Schlotter and P. S. Keogh, "Synchronous position recovery control for flexible rotors in contact with auxiliary bearings," Transactions of the ASME: Journal of Vibration and Acoustics, vol. 129, no. 5, pp. 550-558, 2007.

[11] M. Jang, C. Chen, and Y. Tsao, "Sliding mode control for active magnetic bearing system with flexible rotor," Journal of the Franklin Institute, vol. 342, no. 4, pp. 401-419, 2005.
[12] A. Carrella, M. I. Friswell, A. Zotov, D. J. Ewins, and A. Tichonov, "Using nonlinear springs to reduce the whirling of a rotating shaft," Mechanical Systems and Signal Processing, vol. 23, no. 7, pp. 2228-2235, 2009.

[13] L. Ginzinger and H. Ulbrich, "Control of a rubbing rotor using an active auxiliary bearing," Journal of Mechanical Science and Technology, vol. 21, no. 6, pp. 851-854, 2007.

[14] A. Chavez, H. Ulbrich, and L. Ginzinger, "Reduction of contact forces in a rotor-stator-system in case of rubbing through active auxiliary bearing," Shock and Vibration, vol. 13, no. 4-5, pp. 505518, 2006.

[15] L. Ginzinger and H. Ulbrich, "Simulation-based controller design for an active auxiliary bearing," Journal of System Design and Dynamics, vol. 3, pp. 607-616, 2009.

[16] I. S. Cade, M. N. Sahinkaya, C. R. Burrows, and P. S. Keogh, "An active auxiliary bearing control strategy to reduce the onset of asynchronous periodic contact modes in rotor/magnetic bearing systems," Journal of Engineering for Gas Turbines and Power, vol. 132, no. 5, Article ID 052502, 2010.

[17] S.-M. Wang, Q.-S. Lu, Q. Wang, and P. Xu, "Reducing the amplitude of vibration at resonances by phase modulation," Journal of Sound and Vibration, vol. 290, no. 1-2, pp. 410-424, 2006.

[18] S. Wang, J. Xu, and Q. Wang, "The mechanism of stiffness increase phenomenon of a rubbing disk," Acta Mechanica Sinica, vol. 26, no. 3, pp. 441-448, 2010. 


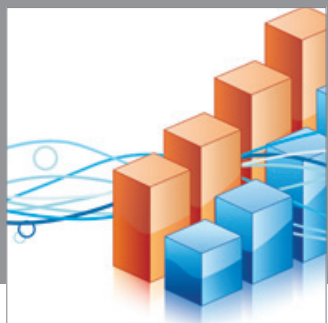

Advances in

Operations Research

mansans

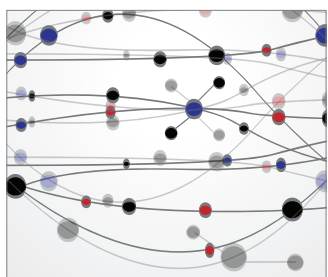

The Scientific World Journal
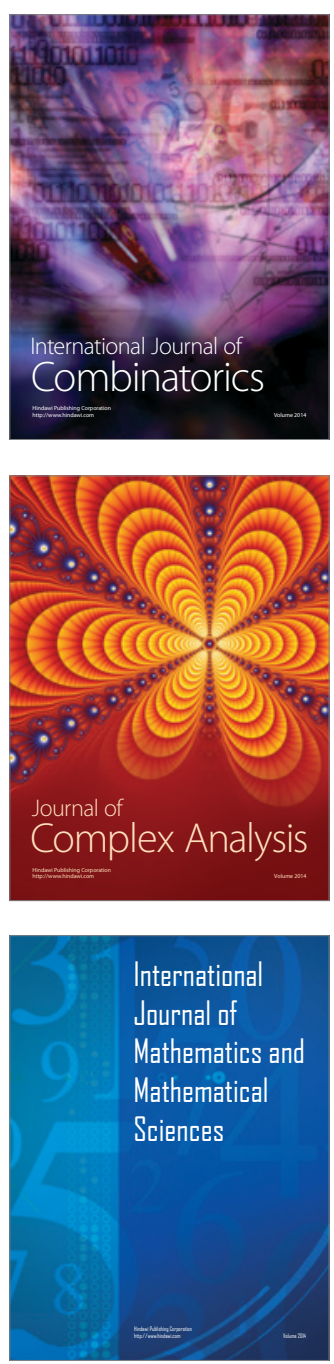
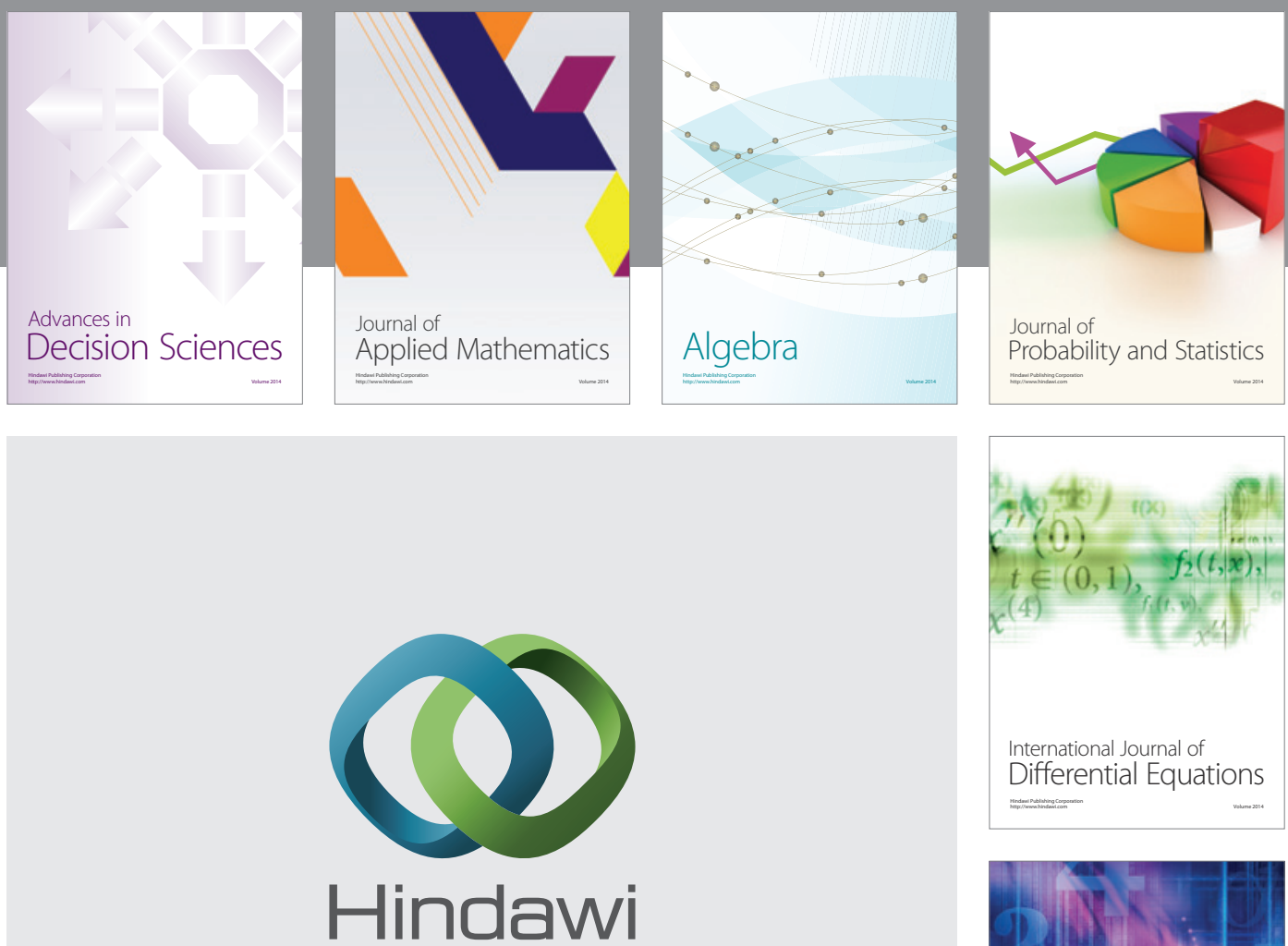

Submit your manuscripts at http://www.hindawi.com
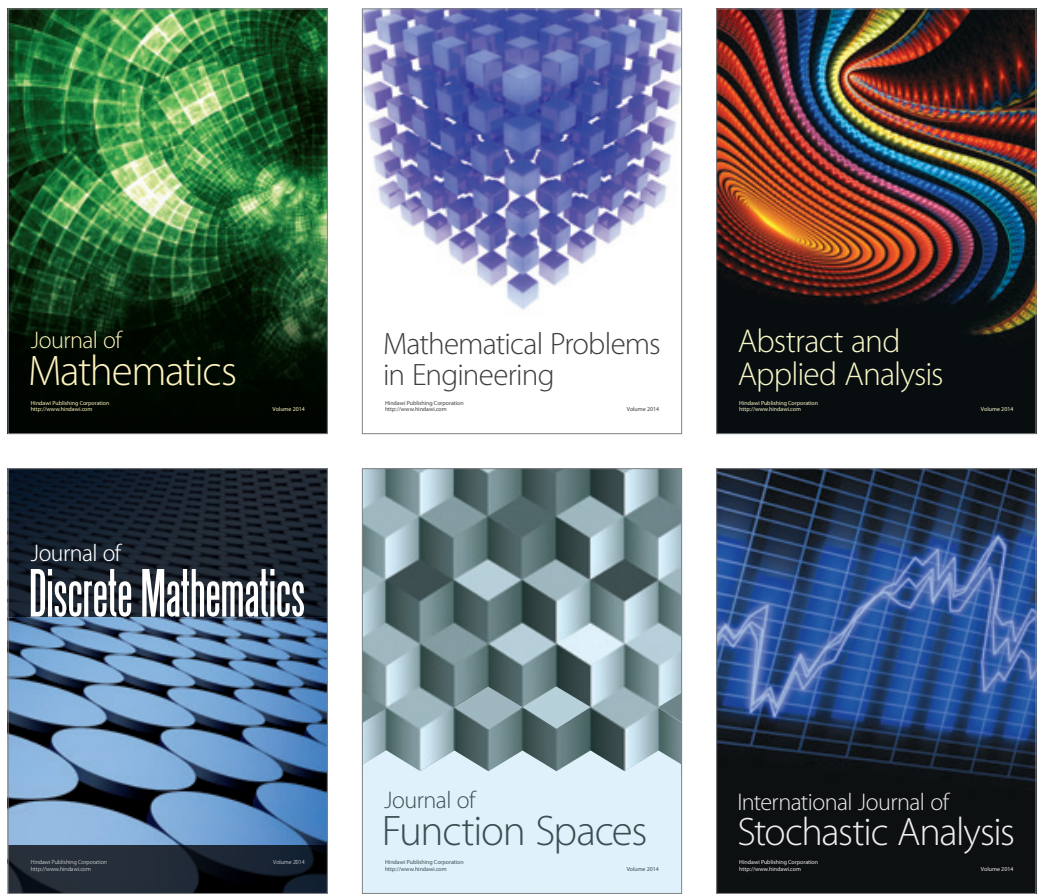

Journal of

Function Spaces

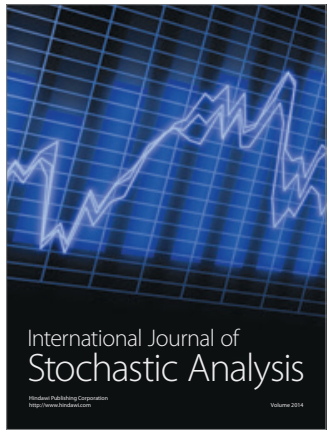

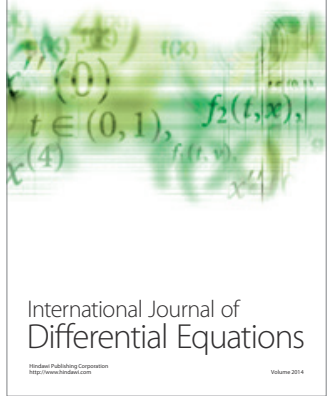
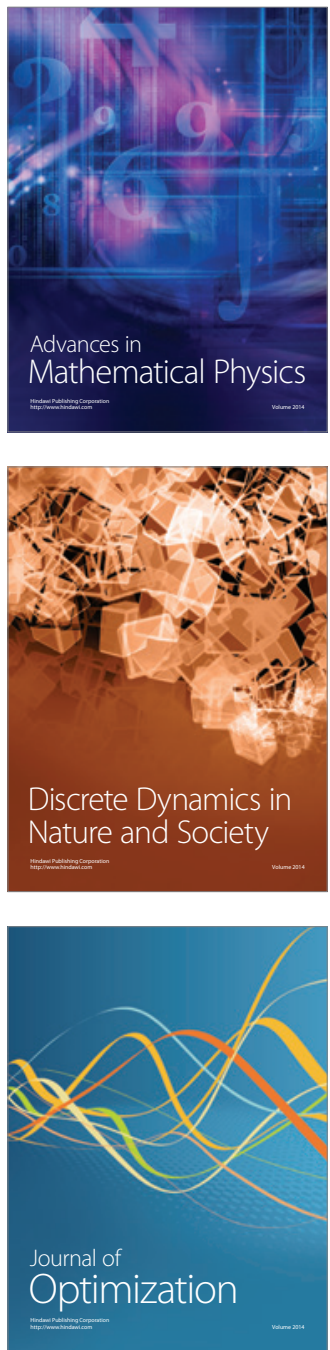